\title{
Ultrasound gel dispensing: There may be more going around than centripetal acceleration
}

\author{
Timothy P. Turkstra, MD
}

Received: 18 May 2015/Accepted: 16 June 2015/Published online: 9 July 2015

(C) Canadian Anesthesiologists' Society 2015

\section{To the Editor,}

I read with interest the letter by Dr. Lecluyse ${ }^{1}$ explaining a technique for more efficient dispensing of ultrasound gel (and other condiments). However interesting the condiment, it is difficult to reconcile the "engineering" explanation, which highlights the challenge in balancing the simplification of complex physics for a nonengineering audience while maintaining some degree of accuracy.

If the acceleration exceeds the limit that the friction forces of the container can exert on the gel (also considering gravity and the suspension effects of the air inside the container), it is correct to reason that the gel will move inside the container, in the desired relative direction if the container is oriented appropriately.

Although an argument could be made to ignore the highly non-linear gel fluid dynamics as well as the distensibility of the container (even though these are reasons for requiring the technique in the first place), further ignoring both gravity and the suspension effect of the air below (and potentially above) the gel portion could

This letter is accompanied by a reply. Please see Can J Anesth 2015; 62: this issue.

Electronic supplementary material The online version of this article (doi:10.1007/s12630-015-0424-x) contains supplementary material, which is available to authorized users.

T. P. Turkstra, MD ( $₫)$

Department of Anesthesia and Perioperative Medicine London Health Sciences Centre, Western University, London, ON, Canada seriously compromise the fidelity of the model. In addition, as both force and acceleration incorporate time to the power of two, the absent time/duration information for this proposed technique is relevant - and could lead to other analyses entirely, including a lack of relevant centripetal acceleration.

Even accepting these simplifications, the author may consider other accelerations that are likely greater than the described centripetal acceleration, particularly as the angular velocity is not constant (available as Electronic Supplementary Material). The angular or lateral acceleration, orthogonal to the radius $r$, is more likely to predominate due to the angular acceleration and deceleration.

Accordingly, the technique may be much more effective if the gel container is rotated substantially with the wrist as well (as partially shown in the original figure). Simply start by pointing the opening of the container upward, then rapidly snap the container downward approximately $1 / 2 \mathrm{~m}$, while rotating the opening downward during this motion, and then stop sharply. With little difficulty, this action will cause $>5 \mathrm{~g}$ of the gel to shift towards the opening.

Anyone attempting this method as a practical example will likely notice the gel moving inside the container at the end of the arc or linear path rather than midway in the angular rotation as a smooth and constant movement - as would be the case if centripetal acceleration predominated. Similarly, feel free to attempt this technique with a cup of water to find out if you can rely only on centripetal acceleration. Unless you rotate your wrist dramatically during this technique, you may be left wet due to the angular acceleration and gravitational component.

Fortunately, just as we do not need to understand variable valve timing, let alone the difference between static and dynamic friction, to drive an automobile safely, 
the technique proposed by Dr. Lecluyse is reasonable even if the "engineering" explanation stretches the limits for enthusiastic simplification.

Conflicts of interest None declared.

\section{Reference}

1. Lecluyse $V$. Understanding the physics and fluid dynamics governing ultrasound gel behaviour facilitates its use: the ketchup analogy! Can J Anesth 2015; 62: 427-8. 